\title{
HARDY-RAMANUJAN JOURNAL
}

(A Journal devoted to primes, diophantine equations, transcendental numbers and other questions on

$1,2,3,4,5, \ldots)$

VOLUME 28

2005

Date of issue: 22.12 .2005

(To be put on the internet around this time)

EDITORS:

R.BALASUBRAMANIAN AND K.RAMACHANDRA 
Hardy Ramanujan Journal

Vol. 28(2005) 1-9

\title{
Further Variations on the Six Exponentials Theorem
}

\author{
Michel Waldschmidt
}

\begin{abstract}
Let $\widetilde{\mathcal{L}}$ denote the set of linear combinations, with algebraic coefficients, of 1 and logarithms of algebraic numbers. The Strong Six Exponentials Theorem of D. Roy gives sufficient conditions for a $2 \times 3$ matrix

$$
M=\left(\begin{array}{lll}
\Lambda_{11} & \Lambda_{12} & \Lambda_{13} \\
\Lambda_{21} & \Lambda_{22} & \Lambda_{23}
\end{array}\right)
$$
\end{abstract}

whose entries are in $\widetilde{\mathcal{L}}$ to have rank 2 .

Here we give sufficient conditions so that one at least of the three $2 \times 2$ determinants

is not in $\widetilde{\mathcal{L}}$

$$
\left|\begin{array}{ll}
\Lambda_{11} & \Lambda_{12} \\
\Lambda_{21} & \Lambda_{22}
\end{array}\right|, \quad\left|\begin{array}{ll}
\Lambda_{12} & \Lambda_{13} \\
\Lambda_{22} & \Lambda_{23}
\end{array}\right|, \quad\left|\begin{array}{ll}
\Lambda_{13} & \Lambda_{11} \\
\Lambda_{23} & \Lambda_{21}
\end{array}\right|
$$

\section{Main result}

We denote by $\mathbb{Q}$ the field of rational numbers, by $\overline{\mathbb{Q}}$ the field of algebraic numbers (algebraic closure of $\mathbb{Q}$ in $\mathbb{C}$ ), by $\mathcal{L}$ the $\mathbb{Q}$-vector space of logarithms of algebraic numbers:

$$
\mathcal{L}=\left\{\lambda \in \mathbb{C} ; e^{\lambda} \in \overline{\mathbb{Q}}^{\times}\right\}=\left\{\log \alpha ; \alpha \in \overline{\mathbb{Q}}^{\times}\right\}=\exp ^{-1}\left(\overline{\mathbb{Q}}^{\times}\right)
$$

and by $\widetilde{\mathcal{L}}$ the $\overline{\mathbb{Q}}$-vector subspace of $\mathbb{C}$ spanned by $\{1\} \cup \mathcal{L}$. Hence $\widetilde{\mathcal{L}}$ is the set of linear combinations of 1 and logarithms of algebraic numbers with algebraic coefficients:

$$
\begin{aligned}
\widetilde{\mathcal{L}}=\left\{\beta_{0}+\beta_{1} \log \alpha_{1}+\cdots+\beta_{n} \log \alpha_{n}\right. & \\
& \left.n \geq 0,\left(\alpha_{1}, \ldots, \alpha_{n}\right) \in\left(\overline{\mathbb{Q}}^{\times}\right)^{n},\left(\beta_{0}, \beta_{1}, \ldots, \beta_{n}\right) \in \overline{\mathbb{Q}}^{n+1}\right\} .
\end{aligned}
$$

Here is the so-called strong six exponentials Theorem of D. Roy ( ([5] Corollary $2 \S 4$ p. 38; see also [7] Corollary 11.16):

Key words and phrases. Transcendental numbers, logarithms of algebraic numbers, four exponentials Conjecture, six exponentials Theorem, algebraic independence.

Acknowledgements: A suggestion by D. Roy in Banff in november 2004 turned out to be a key point in the proof of the main result. Thanks also to him and to Guy Diaz for their comments on previous versions of this text. 
TheOREM 1.1. Let $M$ be a $2 \times 3$ matrix with entries in $\widetilde{\mathcal{L}}$ :

$$
M=\left(\begin{array}{lll}
\Lambda_{11} & \Lambda_{12} & \Lambda_{13} \\
\Lambda_{21} & \Lambda_{22} & \Lambda_{23}
\end{array}\right)
$$

Assume that the two rows of $M$ are linearly independent over $\overline{\mathbb{Q}}$ and also that the three columns are linearly independent over $\overline{\mathbb{Q}}$. Then $M$ has rank 2 .

Consider the three $2 \times 2$ determinants

$$
\Delta_{1}=\Lambda_{12} \Lambda_{23}-\Lambda_{13} \Lambda_{22}, \Delta_{2}=\Lambda_{13} \Lambda_{21}-\Lambda_{11} \Lambda_{23}, \Delta_{3}=\Lambda_{11} \Lambda_{22}-\Lambda_{12} \Lambda_{21} \text {. }
$$

From the relation

$$
\Delta_{1}\left(\begin{array}{l}
\Lambda_{11} \\
\Lambda_{21}
\end{array}\right)+\Delta_{2}\left(\begin{array}{c}
\Lambda_{12} \\
\Lambda_{22}
\end{array}\right)+\Delta_{3}\left(\begin{array}{c}
\Lambda_{13} \\
\Lambda_{23}
\end{array}\right)=0
$$

it follows from the assumptions of Theorem 1.1 that one at least of the three numbers $\Delta_{1}, \Delta_{2}, \Delta_{3}$ is transcendental. We want to prove that one at least of these three numbers is not in $\widetilde{\mathcal{L}}$.

If the five rows of the matrix $\left(\begin{array}{c}M \\ I_{3}\end{array}\right)$ (where $I_{3}$ is the $3 \times 3$ identity matrix) are linearly dependent over $\overline{\mathbb{Q}}$, which means that there exists $\left(\gamma_{1}, \gamma_{2}\right) \in$ $\overline{\mathbb{Q}}^{2} \backslash\{0\}$ such that the three numbers

$$
\delta_{j}=\gamma_{1} \Lambda_{1 j}+\gamma_{2} \Lambda_{2 j} \quad(j=1,2,3)
$$

are algebraic, then the three numbers $\Delta_{1}, \Delta_{2}, \Delta_{3}$ are in $\widetilde{\mathcal{L}}$. Indeed, if $(j, h, k)$ denotes any of the triples $(1,2,3),(2,3,1),(3,1,2)$, then

$$
\gamma_{1} \Delta_{j}=\delta_{h} \Lambda_{2 k}-\delta_{k} \Lambda_{2 h} \quad \text { and } \quad \gamma_{2} \Delta_{j}=\delta_{k} \Lambda_{1 h}-\delta_{h} \Lambda_{1 k}
$$

Here is the main result of this paper.

TheOREM 1.2. Let $M$ be a $2 \times 3$ matrix with entries in $\widetilde{\mathcal{L}}$ :

$$
M=\left(\begin{array}{lll}
\Lambda_{11} & \Lambda_{12} & \Lambda_{13} \\
\Lambda_{21} & \Lambda_{22} & \Lambda_{23}
\end{array}\right)
$$

Assume that the five rows of the matrix

$$
\left(\begin{array}{c}
M \\
I_{3}
\end{array}\right)=\left(\begin{array}{ccc}
\Lambda_{11} & \Lambda_{12} & \Lambda_{13} \\
\Lambda_{21} & \Lambda_{22} & \Lambda_{23} \\
1 & 0 & 0 \\
0 & 1 & 0 \\
0 & 0 & 1
\end{array}\right)
$$


are linearly independent over $\overline{\mathbb{Q}}$ and that the five columns of the matrix

$$
\left(I_{2}, M\right)=\left(\begin{array}{ccccc}
1 & 0 & \Lambda_{11} & \Lambda_{12} & \Lambda_{13} \\
0 & 1 & \Lambda_{21} & \Lambda_{22} & \Lambda_{23}
\end{array}\right)
$$

are linearly independent over $\overline{\mathbb{Q}}$. Then one at least of the three numbers

$$
\Delta_{1}=\left|\begin{array}{ll}
\Lambda_{12} & \Lambda_{13} \\
\Lambda_{22} & \Lambda_{23}
\end{array}\right|, \quad \Delta_{2}=\left|\begin{array}{ll}
\Lambda_{13} & \Lambda_{11} \\
\Lambda_{23} & \Lambda_{21}
\end{array}\right|, \quad \Delta_{3}=\left|\begin{array}{ll}
\Lambda_{11} & \Lambda_{12} \\
\Lambda_{21} & \Lambda_{22}
\end{array}\right|
$$

is not in $\widetilde{\mathcal{L}}$.

If $M$ is $d \times \ell$ matrix of rank 1 , with $d \geq 2$ and $\ell \geq 2$, whose columns are $\overline{\mathbb{Q}}$-linearly independent, then the $d+\ell$ columns of the matrix $\left(I_{d} M\right)$ are also $\overline{\mathbb{Q}}$-linearly independent. Hence on the one hand Theorem 1.2 generalizes Theorem 1.1. On the other hand, as noticed by G. Diaz, when one of the six numbers $\Lambda_{i j}$ is algebraic, Theorem 1.2 reduces to the next consequence of Theorem 1.1 (further related results are given in [1] and [8]).

Corollary 1.3. Let $\Lambda_{1}, \Lambda_{2}, \Lambda_{3}$ be three elements of $\widetilde{\mathcal{L}}$ Assume that $\Lambda_{1}$ is transcendental and that the three numbers $1, \Lambda_{2}, \Lambda_{3}$ are $\overline{\mathbb{Q}}$-linearly independent. Then one at least of the two numbers $\Lambda_{1} \Lambda_{2}, \Lambda_{1} \Lambda_{3}$ is not in $\widetilde{\mathcal{L}}$.

The simple example

$$
M=\left(\begin{array}{ccc}
0 & \Lambda_{2} & \Lambda_{3} \\
\Lambda_{1} & 0 & 0
\end{array}\right)
$$

shows that the assumptions of Theorem 1.2 are not sufficient to ensure that none of the three determinants is in $\widetilde{\mathcal{L}}$.

Here is a simple result which follows from Theorem 1.2: Let $\Lambda_{1}, \Lambda_{2}, \Lambda_{3}$ be three elements in $\widetilde{\mathcal{L}}$ such that $1, \Lambda_{1}, \Lambda_{2}, \Lambda_{3}$ are linearly independent over $\overline{\mathbb{Q}}$. Then one at least of the three numbers

$$
\Lambda_{1}^{2}-\Lambda_{2} \Lambda_{3}, \quad \Lambda_{2}^{2}-\Lambda_{3} \Lambda_{1}, \quad \Lambda_{3}^{2}-\Lambda_{1} \Lambda_{2}
$$

is not in $\widetilde{\mathcal{L}}$.

In $\S 3$ we shall deduce from Theorem 1.2 the following corollary.

Corollary 1.4. Let $M$ be a $2 \times 3$ matrix with entries in $\mathcal{L}$ :

$$
M=\left(\begin{array}{ccc}
\lambda_{11} & \lambda_{12} & \lambda_{13} \\
\lambda_{21} & \lambda_{22} & \lambda_{23}
\end{array}\right)
$$

Assume that the two rows of $M$ are linearly independent over $\mathbb{Q}$ and also that the three columns of $M$ are linearly independent over $\mathbb{Q}$. Then one at 
least of the three numbers

$$
\lambda_{11} \lambda_{22}-\lambda_{12} \lambda_{21}, \quad \lambda_{12} \lambda_{23}-\lambda_{13} \lambda_{22}, \quad \lambda_{13} \lambda_{21}-\lambda_{11} \lambda_{23}
$$

is not in $\widetilde{\mathcal{L}}$.

The six exponentials Theorem of S. Lang ([3], Chap. II $\S 1$ ) and K. Ramachandra ([4] II $\S 4$ ) states that, under the assumptions of Corollary 1.4, one at least of the three numbers (1.5) is not zero.

It is expected that a result similar to Theorem 1.2 holds when $M$ is replaced by a $2 \times 2$ matrix:

CONJeCture 1.6. Let $M$ be a $2 \times 2$ matrix with entries in $\widetilde{\mathcal{L}}$ :

$$
M=\left(\begin{array}{ll}
\Lambda_{11} & \Lambda_{12} \\
\Lambda_{21} & \Lambda_{22}
\end{array}\right) \text {. }
$$

Assume that the four rows of the matrix

$$
\left(\begin{array}{c}
M \\
I_{2}
\end{array}\right)=\left(\begin{array}{cc}
\Lambda_{11} & \Lambda_{12} \\
\Lambda_{21} & \Lambda_{22} \\
1 & 0 \\
0 & 1
\end{array}\right)
$$

are linearly independent over $\overline{\mathbb{Q}}$ and that the four columns of the matrix

$$
\left(I_{2}, M\right)=\left(\begin{array}{llll}
1 & 0 & \Lambda_{11} & \Lambda_{12} \\
0 & 1 & \Lambda_{21} & \Lambda_{22}
\end{array}\right)
$$

are linearly independent over $\overline{\mathbb{Q}}$. Then the number

$$
\Delta=\left|\begin{array}{ll}
\Lambda_{11} & \Lambda_{12} \\
\Lambda_{21} & \Lambda_{22}
\end{array}\right|
$$

is not in $\widetilde{\mathcal{L}}$.

Conjecture 1.6 follows from the conjecture (see for instance [3], Historical Note of Chapter III, [2], Chap. 6 p. 259 and [7], Conjecture 1.15 and [8] Conjecture 1.1) that $\mathbb{Q}$-linearly independent logarithms of algebraic numbers are algebraically independent.

\section{A consequence of the Linear Subgroup Theorem}

Let $n$ be a positive integer and $Y$ a $\overline{\mathbb{Q}}$-vector subspace of $\mathbb{C}^{n}$. We define

$$
\mu\left(Y, \mathbb{C}^{n}\right)=\min _{V \subset \mathbb{C}^{n}} \frac{\operatorname{dim}_{\overline{\mathbb{Q}}}(Y / Y \cap V)}{\operatorname{dim}_{\mathbb{C}}\left(\mathbb{C}^{n} / V\right)},
$$

where $V$ runs over the set of $\mathbb{C}$-vector subspaces of $\mathbb{C}^{n}$ with $V \neq \mathbb{C}^{n}$. 
For $x=\left(x_{1}, \ldots, x_{n}\right) \in \mathbb{C}^{n}$ and $y=\left(y_{1}, \ldots, y_{n}\right) \in \mathbb{C}^{n}$ we denote by $x \cdot y$ the scalar product

$$
x \cdot y=x_{1} y_{1}+\cdots+x_{n} y_{n} .
$$

For $X$ and $Y$ two subsets of $\mathbb{C}^{n}$, we denote by $X \cdot Y$ the set of scalar products $x \cdot y$ where $x$ ranges over the set $X$ and $y$ over $Y$.

Theorem 2.1. Let $X$ and $Y$ be two $\overline{\mathbb{Q}}$-vector subspaces of $\mathbb{C}^{n}$. Assume $X$ has dimension $d$ with $d>n$. Assume further

$$
\mu\left(Y, \mathbb{C}^{n}\right)>\frac{d}{d-n} .
$$

Then the set $X \cdot Y$ is not contained in $\widetilde{\mathcal{L}}$.

Proof. This is essentially Proposition 6.1 of $[6]$, where $\mathbb{Q}$ is replaced by $\overline{\mathbb{Q}}$ and the $\mathbb{Q}$-vector space $\mathcal{L}$ by the $\overline{\mathbb{Q}}$-vector space $\widetilde{\mathcal{L}}$. Henceforth the proof runs as follows.

Like in Lemme 5.2 of [6], one checks that if $X$ and $Y$ are two vector subspaces of $\mathbb{C}^{n}$ over $\overline{\mathbb{Q}}$, of dimensions $d$ and $\ell$ respectively, then there exist a positive integer $n^{\prime} \leq n$ and two vector subspaces $X^{\prime}$ and $Y^{\prime}$ of $\mathbb{C}^{n^{\prime}}$, of dimensions $d^{\prime}$ and $\ell^{\prime}$ respectively, such that

$$
\mu\left(X^{\prime}, \mathbb{C}^{n^{\prime}}\right)=\frac{d^{\prime}}{n^{\prime}} \geq \frac{d}{n}, \quad \mu\left(Y^{\prime}, \mathbb{C}^{n^{\prime}}\right)=\frac{\ell^{\prime}}{n^{\prime}} \geq \mu\left(Y, \mathbb{C}^{n}\right)
$$

and

$$
X^{\prime} \cdot Y^{\prime} \subset X \cdot Y
$$

This shows that for the proof of Theorem 2.1, there is no loss of generality to assume $\mu\left(X, \mathbb{C}^{n}\right)=d / n$ and $\mu\left(Y, \mathbb{C}^{n}\right)=\ell / n$. The assumption $\mu\left(Y, \mathbb{C}^{n}\right)>$ $d /(d-n)$ reduces to $\ell d>n(\ell+d)$.

Following the argument of Lemme 5.4 in [6], one proves that if $X$ and $Y$ are two vector subspaces of $\mathbb{C}^{n}$ over $\overline{\mathbb{Q}}$, of dimensions $d$ and $\ell$ respectively, $X_{1}$ a subspace of $X$ of dimension $d_{1}$ and $Y_{1}$ a subspace of $Y$ of dimension $\ell_{1}$ such that $X_{1} \cdot Y_{1}=\{0\}$, then

$$
\left(d-d_{1}\right) \mu\left(Y, \mathbb{C}^{n}\right)+\left(\ell-\ell_{1}\right) \mu\left(X, \mathbb{C}^{n}\right) \geq n \mu\left(X, \mathbb{C}^{n}\right) \mu\left(Y, \mathbb{C}^{n}\right) .
$$

In Lemme 5.4 in [6] an extra assumption is required, namely

$$
\mu\left(X, \mathbb{C}^{n}\right) \mu\left(Y, \mathbb{C}^{n}\right) \geq \mu\left(X, \mathbb{C}^{n}\right)+\mu\left(Y, \mathbb{C}^{n}\right),
$$

but we do not need it here, since our assumption $X_{1} \cdot Y_{1}=\{0\}$ is stronger than the assumption in Lemme 5.4 of $[6]$ that $X_{1} \cdot Y_{1}$ has rank $\leq 1$. 
Next we introduce the coefficient $\theta(M)$ attached to a $d \times \ell$ matrix $M$ with entries in $\mathbb{C}$. It is defined as follows:

$$
\theta(M)=\min \frac{\ell^{\prime}}{d^{\prime}}
$$

where $\left(d^{\prime}, \ell^{\prime}\right)$ ranges over the set of pairs of integers satisfying $0 \leq \ell^{\prime} \leq \ell$, $1 \leq d^{\prime} \leq d$, such that there exist a $d \times d$ regular matrix $P$ and a regular $\ell \times \ell$ regular matrix $Q$, both with entries in $\overline{\mathbb{Q}}$, with

$$
P M Q=\underbrace{\left(\begin{array}{cc}
A & B \\
C & 0
\end{array}\right)}_{\ell^{\prime}} \underbrace{\} d^{*}}_{\ell^{*}}\} d^{\prime}
$$

From (2.3) with $d_{1}=d^{\prime}$ and $\ell_{1}=\ell^{*}$ it follows that if

$$
X=\overline{\mathbb{Q}} x_{1}+\cdots+\overline{\mathbb{Q}} x_{d} \quad \text { and } \quad Y=\overline{\mathbb{Q}} y_{1}+\cdots+\overline{\mathbb{Q}} y_{\ell}
$$

are again two vector subspaces of $\mathbb{C}^{n}$ over $\overline{\mathbb{Q}}$, of dimensions $d$ and $\ell$ respectively, satisfying $\mu\left(X, \mathbb{C}^{n}\right)=d / n$, then the matrix

$$
M=\left(x_{i} \cdot y_{j}\right)_{1 \leq i \leq d, 1 \leq j \leq \ell}
$$

has

$$
\theta(M) \geq \frac{n}{d} \cdot \mu\left(Y, \mathbb{C}^{n}\right)
$$

In particular if $\mu\left(X, \mathbb{C}^{n}\right)=d / n$ and $\mu\left(Y, \mathbb{C}^{n}\right)=\ell / n$, then $\theta(M)=\ell / d$. Finally Theorem 4 in [5] (which is Proposition 11.19 or Theorem 12.19 in [7]) shows that the rank $r$ of a $d \times \ell$ matrix $M$ with entries in $\widetilde{\mathcal{L}}$ satisfies

$$
r \geq \frac{d \theta}{1+\theta}
$$

where $\theta=\theta(M)$. Using this result for the matrix $M$ given by (2.4) whose rank $r$ is $\leq n$, one concludes that if $\mu\left(X, \mathbb{C}^{n}\right)=d / n$ and $\mu\left(Y, \mathbb{C}^{n}\right)=\ell / n$ with $X \cdot Y \subset \widetilde{\mathcal{L}}$, then

$$
n \geq \frac{\ell d}{\ell+d}
$$

Theorem 2.1 follows.

REMARK. Theorem 1.1 is equivalent with the case $n=1$ of Theorem 2.1 . 


\section{Proof of the main results}

In this section we prove Theorem 1.2 and Corollary 1.4.

Proof of Theorem 1.2. Assume that the hypotheses of Theorem 1.2 are satisfied. Define elements $v_{1}, \ldots, v_{5}$ in $\widetilde{\mathcal{L}}^{2}$ by

$$
v_{1}=e_{1}, \quad v_{2}=e_{2}, \quad v_{2+j}=\left(\Lambda_{1 j}, \Lambda_{2 j}\right), \quad(j=1,2,3),
$$

where $e_{1}=(1,0)$ and $e_{2}=(0,1)$. For $v=(x, y) \in \mathbb{C}^{2}$, set $v^{\prime}=(-y, x)$, so that $v^{\prime} \cdot v=0$. Consider the $5 \times 5$ matrix

$$
A=\left(v_{i}^{\prime} \cdot v_{j}\right)_{1 \leq i, j \leq 5} .
$$

From its very definition, it is plain that $A$ has rank 2 . Explicitly one has

$$
A=\left(\begin{array}{ccccc}
0 & 1 & \Lambda_{21} & \Lambda_{22} & \Lambda_{23} \\
-1 & 0 & -\Lambda_{11} & -\Lambda_{12} & -\Lambda_{13} \\
-\Lambda_{21} & \Lambda_{11} & 0 & \Delta_{3} & -\Delta_{2} \\
-\Lambda_{22} & \Lambda_{12} & -\Delta_{3} & 0 & \Delta_{1} \\
-\Lambda_{23} & \Lambda_{13} & \Delta_{2} & -\Delta_{1} & 0
\end{array}\right)
$$

Let $X$ be the $\overline{\mathbb{Q}}$-vector space spanned by $v_{1}, \ldots, v_{5}$ in $\mathbb{C}^{2}$ and similarly let $Y$ be the subspace of $\mathbb{C}^{2}$ spanned by $v_{1}^{\prime}, \ldots, v_{5}^{\prime}$ over $\overline{\mathbb{Q}}$. We claim

$$
\mu\left(X, \mathbb{C}^{2}\right)=\mu\left(Y, \mathbb{C}^{2}\right) \geq 2 .
$$

The equality $\mu\left(X, \mathbb{C}^{2}\right)=\mu\left(Y, \mathbb{C}^{2}\right)$ follows from the fact that the map $(x, y) \mapsto$ $(-y, x)$ is an automorphism of $\mathbb{C}^{2}$.

Since the five columns of $\left(I_{2} \quad M\right)$ are linearly independent over $\overline{\mathbb{Q}}$, $\operatorname{dim}_{\overline{\mathbb{Q}}} X=5$.

Let $V$ is a vector subspace of $\mathbb{C}^{2}$ of dimension 1 and let $t_{1} z_{1}+t_{2} z_{2}=0$ be an equation of $V$ in $\mathbb{C}^{2}$, with $\left(t_{1}, t_{2}\right) \in \mathbb{C}^{2} \backslash\{0\}$. Consider the linear map

$$
p: \begin{array}{ccc}
\mathbb{C}^{2} & \rightarrow & \mathbb{C} \\
\left(z_{1}, z_{2}\right) & \mapsto & t_{1} z_{1}+t_{2} z_{2}
\end{array}
$$

whose kernel is $V$. Since the five rows of $\left(\begin{array}{c}M \\ I_{3}\end{array}\right)$ are $\overline{\mathbb{Q}}$-linearly independent,

$$
\operatorname{dim}_{\overline{\mathbb{Q}}}((X \cap V) / V)=\operatorname{dim}_{\overline{\mathbb{Q}}} p(X) \geq 2 .
$$

This completes the proof of (3.1).

From (3.1) we deduce that the hypothesis $\mu\left(Y, \mathbb{C}^{2}\right)>d /(d-n)$ of Theorem 2.1 is satisfied with $d=5$ and $n=2$, hence the set $X \cdot Y$ is not contained in $\widetilde{\mathcal{L}}$. Consequently one at least of the three numbers $\Delta_{1}, \Delta_{2}, \Delta_{3}$ is not in $\widetilde{\mathcal{L}}$. 
This completes the proof of the Main Theorem 1.2.

REMARK. In (3.1) we may have equality: for instance if $\Lambda_{22}=\Lambda_{23}=0$ then $\mu\left(X, \mathbb{C}^{2}\right)=\mu\left(Y, \mathbb{C}^{2}\right)=2$.

However the proof of Theorem 2.1 shows that in the case $\mu\left(X, \mathbb{C}^{2}\right)=$ $\mu\left(Y, \mathbb{C}^{2}\right)<5 / 2$, Theorem 1.2 should follow from Theorem 1.1. Indeed after a change of variables rational over $\overline{\mathbb{Q}}$ one needs only to consider a matrix

$$
M=\left(\begin{array}{ccc}
0 & \Lambda_{12} & \Lambda_{13} \\
\Lambda_{21} & 0 & 0
\end{array}\right)
$$

which is the situation of Corollary 1.3. If $X$ is the $\overline{\mathbb{Q}}$-subspace of $\mathbb{C}^{2}$ spanned by

$$
v_{1}=(1,0), \quad v_{2}=(0,1), \quad v_{3}=\left(0, \Lambda_{21}\right), \quad v_{4}=\left(\Lambda_{12}, 0\right), \quad v_{4}=\left(\Lambda_{13}, 0\right)
$$

and $Y$ the subspace spanned by

$$
v_{1}^{\prime}=(0,1), v_{2}^{\prime}=(-1,0), v_{3}^{\prime}=\left(-\Lambda_{21}, 0\right), v_{4}^{\prime}=\left(0, \Lambda_{12}\right), v_{4}^{\prime}=\left(0, \Lambda_{13}\right),
$$

then

$$
X^{\prime}=\overline{\mathbb{Q}}+\overline{\mathbb{Q}} \Lambda_{12}+\overline{\mathbb{Q}} \Lambda_{13} \quad \text { and } \quad Y^{\prime}=\overline{\mathbb{Q}}+\overline{\mathbb{Q}} \Lambda_{21}
$$

are $\overline{\mathbb{Q}}$-subspaces of $\mathbb{C}$ satisfying $(2.2)$. Here $\mu\left(X^{\prime}, \mathbb{C}\right)=3>d / n=5 / 2$ and $\mu\left(Y^{\prime}, \mathbb{C}\right)=2=\mu\left(Y, \mathbb{C}^{2}\right)$.

Proof of Corollary 1.4. From Baker's Theorem it follows that if $Y_{0}$ is a $\mathbb{Q}$-vector subspace of $\mathcal{L}^{n}$ of dimension $\ell$, then the $\overline{\mathbb{Q}}$-vector subspace of $\widetilde{\mathcal{L}}^{n}$ spanned by $\overline{\mathbb{Q}}^{n} \cup Y_{0}$ has dimension $\ell+n$ (see Exercise 1.5 (iii) of [7]). Taking firstly $n=2, \ell=3$, and secondly $n=3, \ell=2$, we deduce that the matrix $M$ of corollary 1.4 satisfies the assumptions of Theorem 1.2. Corollary 1.4 follows.

\section{Erratum to [8]}

We take the opportunity of this paper to point out a mistake in the statement of Corollary 2.12 p. 347 of [8]: the assumption that $\Lambda_{21}$ is not zero and $\Lambda_{11} / \Lambda_{21}$ is transcendental should be replaced by the assumption that the three numbers $1, \Lambda_{11}$ and $\Lambda_{21}$ are linearly independent over the field of algebraic numbers. Otherwise a counterexample is obtained for instance with $\Lambda_{21}=1$ and $\Lambda_{2 j}=0$ for $2 \leq j \leq 5$. 


\section{References}

[1] G. Diaz - "Utilisation de la conjugaison complexe dans l'étude de la transcendance de valeurs de la fonction exponentielle", J. Théor. Nombres Bordeaux 16 (2004), p. 535553.

[2] N. I. Fel'd dan \& Y. V. Nesterenko - "Transcendental numbers", in Number theory, IV, Encyclopaedia Math. Sci., vol. 44, Springer, Berlin, 1998, p. 1-345.

[3] S. LANG - Introduction to transcendental numbers, Addison-Wesley Publishing Co., Reading, Mass.-London-Don Mills, Ont., 1966.

[4] K. RamachandRA - "Contributions to the theory of transcendental numbers. I, II", Acta Arith. 14 (1967/68), 65-72; ibid. 14 (1967/1968), p. 73-88.

[5] D. RoY - "Matrices whose coefficients are linear forms in logarithms", J. Number Theory 41 (1992), no. 1, p. 22-47.

[6] M. WALDSChmidT - "Transcendance et exponentielles en plusieurs variables", Invent. Math. 63 (1981), no. 1, p. 97-127.

[7] _ Diophantine approximation on linear algebraic groups, Grundlehren der Mathematischen Wissenschaften [Fundamental Principles of Mathematical Sciences], vol. 326, Springer-Verlag, Berlin, 2000.

[8] _ "Variations on the Six Exponentials Theorem;", Algebra and Number Theory, Proceedings of the Silver Jubilee Conference University of Hyderabad, ed. R. Tandon. Hindustan Book Agency 2005, 338-355. Appendix by Hironori Shiga: "Periods of the Kummer surface;" 356-358.

2000 Mathematics Subject Classification. 11J81 11J86 11J89.

Email address: miw@math.jussieu.fr

URL:http://www.math.jussieu.fr/ miw/ 